\title{
CGCG 480-022: A DISTANT LONESOME MERGER?
}

\author{
C. Carretero, ${ }^{1}$ A. Vazdekis, ${ }^{1}$ A. C. González-García, ${ }^{1}$ J. E. Beckman, ${ }^{1,2}$ and V. Quilis ${ }^{3}$ \\ Received 2006 June 30; accepted 2006 July 28; published 2006 August 24
}

\begin{abstract}
We present a complete analysis, which includes morphology, kinematics, stellar populations, and $N$-body simulations, of CGCG 480-022, the most distant $\left(c z=14,317 \mathrm{~km} \mathrm{~s}^{-1}\right)$ isolated galaxy studied so far in such detail. The results all support the hypothesis that this galaxy has suffered a major merger event with a companion of $\sim 0.1$ times its mass. Morphology reveals the presence of a circumnuclear ring and possibly further ring debris. The radial velocity curve looks symmetrical, while the velocity dispersion increases with radius, reaching values that do not correspond to a virialized system. Moreover, this galaxy deviates significantly from the fundamental plane and the Faber-Jackson relation. The stellar population analysis show that the ring is younger and more metal-rich, which suggest that it has undergone a fairly recent burst of star formation. Both morphological and dynamical results are in broad agreement with our $N$-body simulations.
\end{abstract}

Subject headings: galaxies: abundances — galaxies: individual (CGCG 480-022) — galaxies: interactions — galaxies: kinematics and dynamics - galaxies: structure - methods: $n$-body simulations

\section{INTRODUCTION}

Elliptical galaxies located in very low density environments are highly uncommon but hold important clues about galaxy formation and evolution. For example, current hierarchical models for galaxy formation predict that ellipticals in lowdensity environments have stellar populations that are younger by 1-2 Gyr than their cluster counterparts (e.g., Baugh et al. 1998; Clemens et al. 2006; de Lucia et al. 2006). In this framework, isolated ellipticals are thought to be formed in mergers of galaxy pairs or small groups of galaxies (Jones et al. 2000; D'Onghia et al. 2005). In the first case, the mergers will give rise to kinematical misalignments, while in the second, they will produce an increasing amplitude of rotation with galactocentric radius, as well as rotation around the minor axis (Weil \& Hernquist 1996).

Detailed studies involving morphology, kinematics, and/or stellar populations of isolated ellipticals have been restricted to nearby galaxies $\left(c z<10,000 \mathrm{~km} \mathrm{~s}^{-1}\right.$; e.g., Collobert et al. 2006). In this Letter, we perform an exhaustive analysis of CGCG 480-022 $\left(c z=14,317 \mathrm{~km} \mathrm{~s}^{-1}\right)$, which includes morphological, kinematical, stellar population, and $N$-body simulation properties. We present evidence supporting the hypothesis that this galaxy suffered a major merger event. We adopt a cosmological model with $H_{0}=71 \mathrm{~km} \mathrm{~s}^{-1} \mathrm{Mpc}^{-1}, \Omega_{m}=$ 0.3 , and $\Omega_{\Lambda}=0.7$.

\section{OBSERVATIONS AND DATA REDUCTION}

Optical and near-IR images were extracted from the Digitized Sky Survey (DSS) and Two Micron All Sky Survey (2MASS) catalogs, respectively. Spectroscopy was obtained with the DOLORES spectrograph on the $3.5 \mathrm{~m}$ Telescopio Nazionale Galileo at La Palma (Spain) on 2005 October 3. We used the medium-resolution MR-B 2 grism, in the wavelength range $\lambda \lambda 3500-7000 \AA$, and a $1 " .1 \times 50^{\prime \prime}$ slit. The dispersion was $1.7 \AA$ pixel $^{-1}$ with an instrumental resolution of $8 \AA$ (FWHM).

\footnotetext{
${ }^{1}$ Instituto de Astrofísica de Canarias, Vía Láctea s/n, 38200 La Laguna, Tenerife, Spain; cch@iac.es, vazdekis@iac.es, cglez@iac.es.

${ }^{2}$ Consejo Superior de Investigaciones Científicas, 28006 Madrid, Spain; jeb@iac.es.

${ }^{3}$ Departamento de Astronomía y Astrofísica, Universidad de Valencia, E46100 Burjassot, Valencia, Spain; vicent.quilis@uv.es.
}

We obtained $10 \times 30$ minute exposures, interspersed with $\mathrm{He}+\mathrm{Ar}$ arc-lamp spectra, flux, and radial velocity standards in the same instrumental setup. The seeing was 1 "1.

Data reduction was performed using standard IRAF packages. Once wavelength and flux-calibrated, the spectra were extracted in the spatial direction co-adding 4 pixels to reach the seeing size, or more if necessary to reach a signal-to-noise ratio $\geq 30$ (see Cardiel et al. 1998). Following this procedure, we obtained 13 bins out to $r= \pm 0.8 R_{\text {eff }}$.

\section{ANALYSIS AND RESULTS}

\subsection{Morphology}

To determine morphological and photometric parameters we used the code described in Trujillo et al. (2001) on $K$-band and optical images. Table 1 presents, for each morphological parameter, the average value obtained from both bands. Figure $1 a$ shows the $K$-band image, while Figure $1 b$ shows the optical image with the spectrograph slit overplotted. According to our morphological parameters, as well as its optical appearance, CGCG 480-022 seems to be a typical elliptical galaxy. However, when inspecting the $K$-band image, we detect possible tidal tails or ring debris. In order to search for the existence of more subestructures, we performed a two-dimensional (2D) fit of the galaxy using GALFIT (Peng et al. 2002) with those parameters listed in Table 1 . Figures $1 c$ and $1 d$ show the residuals obtained when subtracting 2D models from each image in $K$ band and optical, respectively. In both cases we clearly observe a welldefined ring at $r \sim 5^{\prime \prime}$, yielding to a rippled structure similar to the shells found in other galaxies (e.g., Quinn 1984). Residuals in the $K$ band are compatible with $b-r$ color contours overplotted on Figure $1 c$.

\subsection{Kinematics}

The radial velocity for each bin was determined by crosscorrelating the observed spectrum with a set of representative synthetic single stellar population spectra, from the model of Vazdekis (1999) . This method is described in Bottema (1988) following the paper of Tonry \& Davis (1979). The measured $V_{r}$ values are represented versus radius in Figure $2 a$. Surprisingly, $V_{r}$ decreases with galactocentric distance at both sides of the center, out of $250 \mathrm{~km} \mathrm{~s}^{-1}$ at $r \sim 3^{\prime \prime}$. We also see a relative 
TABLE 1

Determined Properties of CGCG 480-022

\begin{tabular}{|c|c|}
\hline Parameter & Value \\
\hline$c z\left(\mathrm{~km} \mathrm{~s}^{-1}\right) \ldots \ldots \ldots \ldots \ldots$ & 14,317 \\
\hline$m_{k}(\operatorname{mag}) \ldots \ldots \ldots \ldots \ldots$ & 11.01 \\
\hline$\left\langle\mu_{k}\right\rangle\left(\operatorname{mag} \operatorname{arcsec}^{-2}\right) \ldots \ldots$ & 19.1 \\
\hline$R_{\text {eff }}(\operatorname{arcsec}) \ldots \ldots \ldots \ldots \ldots$ & 13.0 \\
\hline$n \ldots \ldots \ldots \ldots$ & 3.87 \\
\hline$e \ldots \ldots \ldots \ldots$ & 0.1 \\
\hline P.A. $(\mathrm{deg}) \ldots \ldots \ldots \ldots \ldots$ & 89 \\
\hline
\end{tabular}

increase of $100 \mathrm{~km} \mathrm{~s}^{-1}$ where the ring is located. Note that the plotted region represents $0.8 R_{\text {eff }}$.

To measure the velocity dispersion, $\sigma$, we followed the method described in Davies et al. (1993). Figure $2 b$ shows $\sigma$ versus radius. We observe that, within the errors, regions outside the ring present an almost constant $\sigma \sim 400 \mathrm{~km} \mathrm{~s}^{-1}$ while the inner parts look more ordered, i.e., $\sigma$ decreases, reaching $250 \mathrm{~km} \mathrm{~s}^{-1}$ in the center. A similar profile has been found for the isolated massive galaxy NGC 1700 (Bergmann 2002).

\subsection{N-Body Simulations}

To aid our understanding of these results we have performed $\mathrm{N}$-body simulations. Our numerical models simulated a merger between two elliptical galaxies with a mass ratio of $10: 1$ and $5: 1$, and the flyby of a dwarf galaxy about a massive elliptical. As initial conditions for our experiments we used (1) isotropic spherical Jaffe (1983) models for the elliptical galaxies and (2) a King (1966) model for the dwarf galaxy, with a halo modeled with an Evans model. See González-García \& van Albada (2005) for further details of this model and initial conditions for the elliptical galaxies, and Kuijken \& Dubinski (1995) for the dwarf galaxy model.

We used the tree-code GADGET1.1 (Springel et al. 2001) employing $10^{5}$ particles for each model. Low-mass Jaffe models are scaled down homologous systems as the model with mass 1 and with 10 or 5 times fewer particles. Parabolic grazing orbits are employed for the merger simulations, while a hyperbolic encounter is used for the flyby experiment. Energy was well conserved, with errors well below $0.1 \%$.

The material originally belonging to the small galaxy in the $5: 1$ merger finally lies on a fat disklike distribution, while that of the 10:1 merger produces a torus-like body. The flyby encounter produces shells and ripples in the inner parts of the giant galaxy from particles belonging to the satellite; however, no ring or disk is produced in this encounter.

We have obtained a broad estimate of the mass ratio between our target galaxy and a possible companion. If we consider the merger as an inelastic collision, then the whole kinetic energy of the satellite will be transformed into "thermal" energy of the stars of the main galaxy. This process will create a velocity dispersion, $\sigma$, given by $\frac{1}{2} m v_{\text {sat }}^{2}=\frac{1}{2} M \sigma^{2} \Rightarrow M / m=\left(v_{\text {sat }} / \sigma\right)^{2}$, where $v_{\text {sat }}$ is the relative velocity of the satellite with respect to the main galaxy and $M(m)$ is the mass of the central (satellite) galaxy. Considering that they are field galaxies, the proper motion should be of the order of $v_{\text {sat }} \sim 10^{3} \mathrm{~km} \mathrm{~s}^{-1}$, while $\sigma \sim 300 \mathrm{~km} \mathrm{~s}^{-1}$. Then we find $M / m \sim 10$. Moreover, of the models explored, the E+E 10:1 merger is the one with properties closer to those reported for CGCG 480-022. Figure 3 presents the particle distribution of the $10: 1$ system. A ring is clearly observable at a galactocentric distance comparable to that detected in CGCG 480-022. The global kinematics as seen from the same point of view are given in Figure 3. The velocity curve
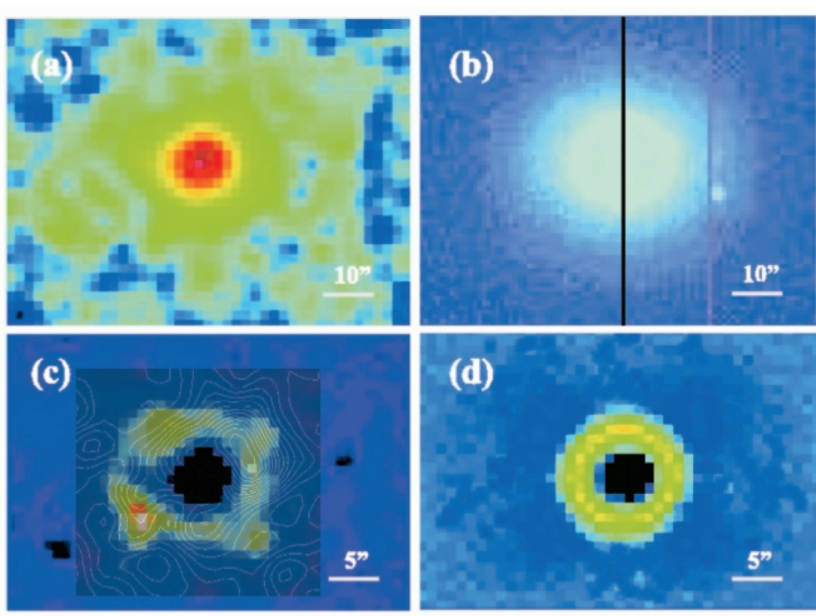

FIG. 1.-Morphology analysis. Panels $a$ and $b$ show the actual images of CGCG 480-022 in the $K$-band and optical, respectively. Panels $c$ and $d$ present the residuals when subtracting a $2 \mathrm{D}$ model of the galaxy from the images using GALFIT. In both cases, a ring of $r \sim 5^{\prime \prime}$ is clearly visible. It is noteworthy that some taillike structures appear in the outer regions of panel $a$. Contours in panel $c$ correspond to $b-r$ color values. The spectrograph slit is overplotted on panel $b$.

presents a drop as we move to larger radii, in agreement with the observations, but the velocity dispersion presents a general declining profile, contrary to what is observed.

\subsection{Stellar Populations}

The stellar population analysis was performed following the prescriptions described in Carretero et al. (2004). To derive mean luminosity-weighted ages and metallicities, we compared selected absorption line strengths with those predicted by the model of Vazdekis (1999). This model provides flux-calibrated

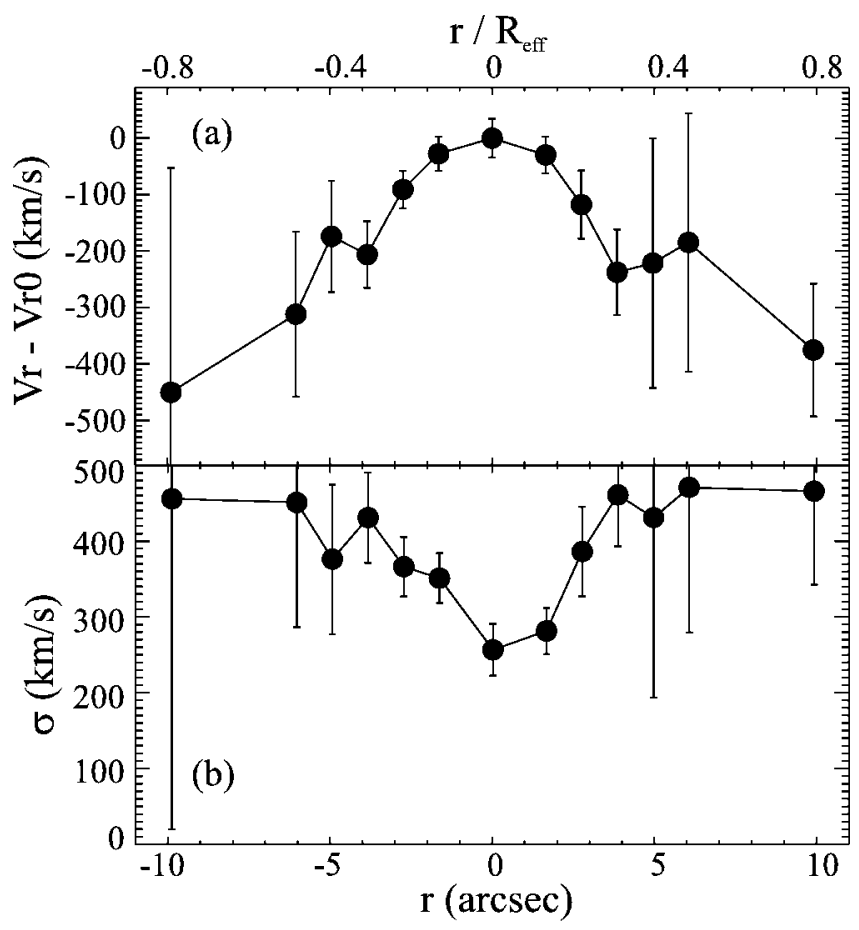

FIG. 2.-Kinematical results. Top: Radial velocity relative to the central value vs. radius. Note that the symmetric shape does not correspond to a typical rotation curve. Bottom: Velocity dispersion vs. radius. 

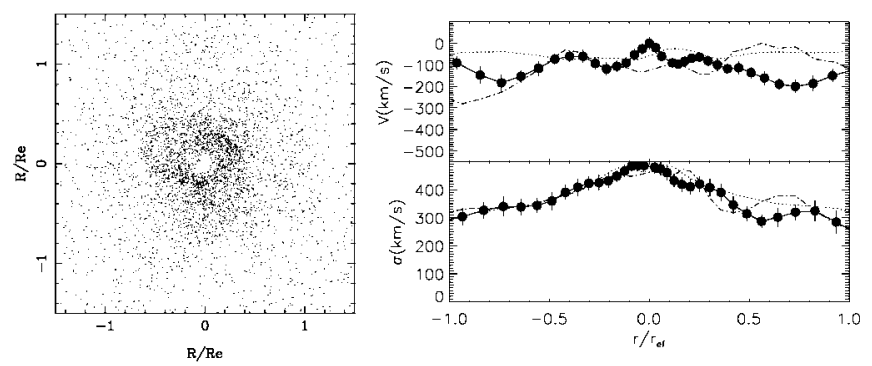

FIG. 3. $-N$-body results. Left: Material from the smaller system in the $10: 1$ merger remnant (for clarity, the large galaxy component is not shown). Note the presence of a well-defined ring at a galactocentric radius similar to that of the observed ring. Right: Kinematics for all the visible material of the merged system. Solid, dashed-dotted and dotted lines correspond to the system at 2, 2.1, and 5 Gyr after the merger, respectively. Top ight: Radial velocity relative to the central value vs. radius. Bottom right: Velocity dispersion vs. radius.

spectra in the optical range at a resolution of $1.8 \AA$ (FWHM) for single-burst stellar populations. This way, we can transform synthetic spectra to the resolution and dispersion of the galaxy spectrum. Plots of the strengths of selected indices $\left(\mathrm{Mg}_{2}\right.$ and Fe4383; Worthey et al. 1994) versus $\mathrm{H} \beta$ provide close to orthogonal model grids, allowing an accurate estimate of galaxy mean age and abundances of the different elements. We have determined mean ages and relative abundances $[\mathrm{Mg} / \mathrm{H}]$ and $[\mathrm{Fe} / \mathrm{H}]$ at each galactocentric distance, computing their radial gradients.

Figure 4 presents the ages and abundances for each element with respect to the central values, versus the radial distance. If we exclude those regions within $3^{\prime \prime}<|r|<6^{\prime \prime}$, we observe clear gradients for both ages and abundances, with the age increasing toward outer regions and abundances decreasing throughout the galactic radius. This result is in agreement with several studies involving gradients in elliptical galaxies (e.g., Davies et al. 1993; Fisher et al. 1995; Kobayashi \& Arimoto 1999).

When considering the region $3^{\prime \prime}<|r|<6^{\prime \prime}$, where the ring is located, we observe a decrease in age with respect to the surrounding regions, associated with a bump in metallicity. This suggests the occurrence of a star formation burst coupled with the presence of the ring.

\section{DISCUSSION}

The morphological, kinematical, $N$-body simulations and stellar population analysis all suggest that CGCG 480-022 could have experienced a major merger event with an alleged companion. In this section, we focus on the evidence provided by each approach.

Morphology.-The residuals obtained when subtracting a 2D model from the images show, in all bands, the presence of a ring of $r \sim 5^{\prime \prime}$. This ring, together with outer streams of material appearing in $K$-band images, could trace the propagating sound waves produced by a merger (e.g., Hernquist \& Quinn 1987) more likely than by the action of tidal interactions or asymmetric star formation ${ }^{4}$ (Colbert et al. 2001). In fact, Malin \& Carter (1983) found that morphological peculiarities, such as shells or ripples, occur roughly 5 times more frequently in environments outside of rich clusters. This result was confirmed by Colbert et al. (2001), who concluded that shells are much more prevalent in isolated galaxies than in group galaxies. Note that the ROSAT

\footnotetext{
${ }^{4}$ Even though we found a star formation burst in the ring, which would be induced by the passing wave; i.e., the varying star formation history should be the effect of the ring structure, not the cause.
}

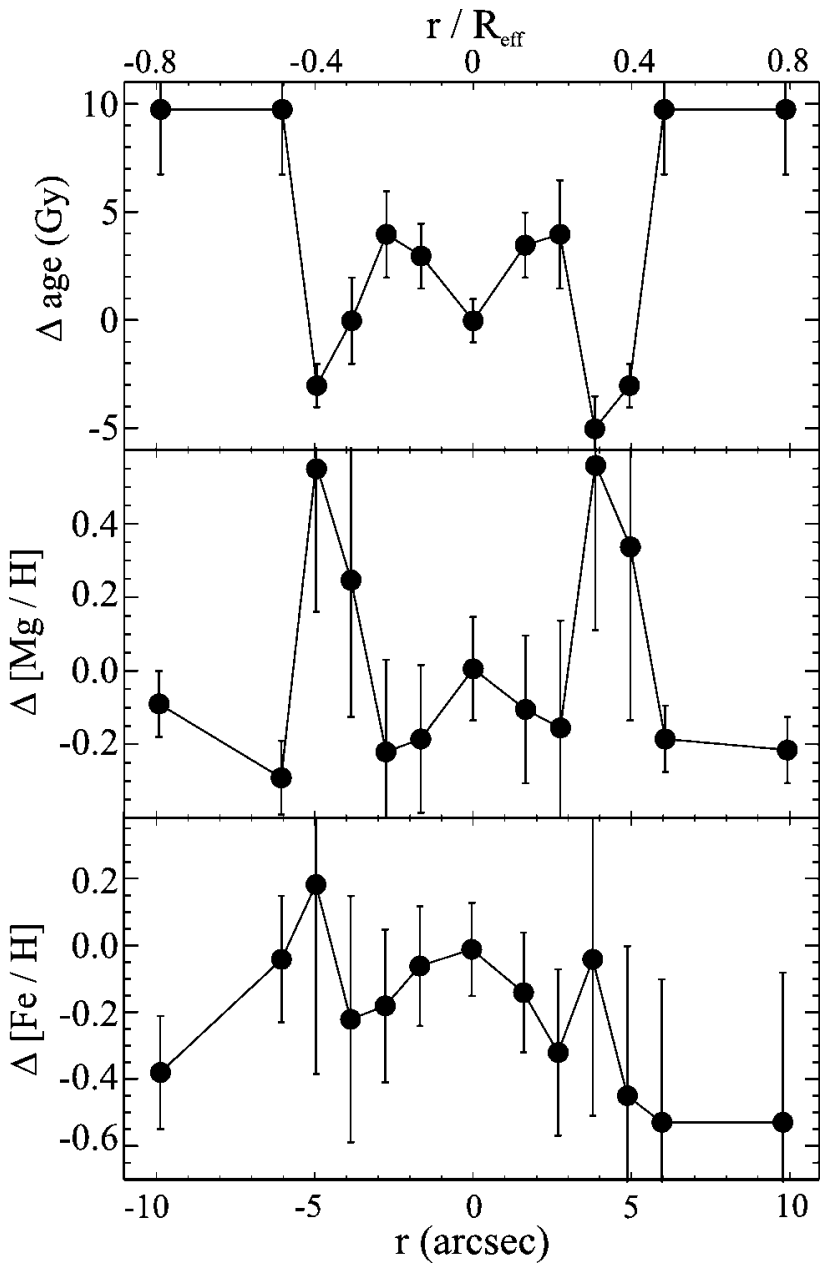

FIG. 4.-Results of the stellar population analysis. The plots show the values of the estimated ages and abundances of individual elements, relative to the center. Expected radial gradients for both ages and metallicities are obtained if we exclude the region $3^{\prime \prime}<|r|<6^{\prime \prime}$. At radial distances corresponding to the position of the ring, stellar population analysis indicates the presence of a recent star formation burst, with younger and more metal-rich stars.

X-ray image of the galaxy (Zimmermann et al. 2001) shows two symmetric lobes in the north-south direction.

Kinematics.-The most intriguing of our results concerns the kinematics of the galaxy. First, within the errors, we find a constant $\sigma \sim 400 \mathrm{~km} \mathrm{~s}^{-1}$, which decreases in the interior of the ring. A similar pattern has been found in other isolated massive galaxy (NGC 1700; Bergmann 2002). However, such high $\sigma$ does not correspond to a virialized system, but in a merger scenario, the intrinsic velocity dispersion should be affected by the collision, yielding higher values of $\sigma$. Second, the radial velocity curve looks symmetrical. Furthermore, both $\sigma$ and $V_{r}$ values bump at the position where the ring is located, i.e., $3^{\prime \prime}<|r|<6^{\prime \prime}$. As an additional test, we checked whether CGCG 480-022 follows the fundamental plane (FP; Djorgovski \& Davis 1987) and the Faber-Jackson (Faber \& Jackson 1976), the Kormendy (Kormendy 1977), and the $\mathrm{Mg}_{2}-\sigma$ (Terlevich et al. 1981) relations. We considered these relations in the near-IR, with data taken from Pahre et al. (1998). CGCG 480-022 deviates $3 \sigma$ from the FP, $6 \sigma$ from the Faber-Jackson relation, and $4 \sigma$ from the $\mathrm{Mg}_{2}-\sigma$ relation, but just $0.2 \sigma$ from the Kormendy relation. Our Galaxy is an outlier in those relations involving the velocity dispersion, in the sense that it should be lower if it corresponds to the intrinsic velocity dis- 
persion of a virialized system. Thus, a major event affecting the dynamics of this galaxy must have occurred. A similar explanation could be proposed for the $V_{r}$ curve, which does not correspond to a rotating system. In order to disentangle the kinematical peculiarities found in this galaxy, future 2D spectroscopy will be extremely helpful, especially to check if our derived kinematics depend on the slit direction.

$N$-body simulations. - The $N$-body experiments performed suggest that a possible formation mechanism is a merger between two elliptical galaxies with a mass ratio near $10: 1$, and meeting each other on a grazing orbit. In such an encounter the smaller system spirals inward while the merger is proceeding finally into a torus-like structure. Such a torus as seen face-on resembles a ring, and the kinematics imposed to the overall field would present a discontinuity. A significant success for this model is that it reproduces without finetuning the velocity profile observed in CGCG 480-022. The central zones move with a larger velocity than the zones at increasing galactocentric distances, with respect to the systemic velocity of the galaxy, and there are secondary maxima at the radial location of the ring in both directions from the center. The range of values of the velocity dispersion predicted by the model is commensurate with that observed, but the decline in value as the center is approached is not reproduced. A reasonable explanation for this is that more ordered motion near the galaxy center could result from an inner disk within the ring radius. The present merger simulation has not, for simplicity, included the presence of gas, but merger models that do include gas (e.g., Barnes 2002) show a tendency for the gas to fall toward the center of the galaxy and form a disk. Also, more detailed $\mathrm{N}$-body $+\mathrm{SPH}$ simulations including star formation and feedback processes will help one to better understand the central kinematics of the system.

Stellar populations.-Following our merger hypothesis, there should be a star formation burst in the ring due to the passing sound wave. Therefore, the stars in the ring should be younger and more metal-rich than those within inner regions, as we found. Given that we know the geometry of the ring, we can broadly estimate the crossing time of the sound wave through the position of the ring, under the following assumptions: (1) the sound speed is constant throughout the whole system and (2) the system behaves as an ideal gas. Then the mean pressure of a system of particles with a given velocity dispersion, $\sigma$, is $p=\frac{1}{3} \rho \sigma^{2}$. The propagation velocity of the perturbation corresponds to the sound speed, $c_{s}$, as $c_{s}^{2}=(d p / d \rho)=\frac{1}{3} \sigma^{2} \Rightarrow c_{s} \sim 180 \mathrm{~km} \mathrm{~s}^{-1}$. Considering that the ring radius is $\sim 5^{\prime \prime}$, the elapsed time since the formation of the perturbation is $t(s)=4.8 \times 10^{-6} \mathrm{D} / c_{s}$, where $D$ is the distance to the galaxy in units of kilometers. Therefore, $t$ should be the stellar age difference between the ring and the center. Given that $D=6.2 \times 10^{23} \mathrm{~km}$, then $t=5.3 \mathrm{Gyr}$, which is in good agreement with our stellar population results (see Fig. 4). Even though approximative, these numbers do support the merger hypothesis.

A question we would like to pose is why the most distant isolated elliptical galaxy so far studied in such detail looks so peculiar. To confirm the results presented here and probe the origin of this system, deep 2D spectroscopy is strongly needed. We also note that CGCG 480-022 is not a completely isolated galaxy but has one confirmed companion at a distance of $0.25 \mathrm{Mpc}$ : PGC 003298 (Beers et al. 1983). The DSS image of this galaxy shows an elongated shape in the direction of CGCG 480-022, while $J$ and $K$-band 2MASS images present some taillike structures. Will PGC 003298 be the next to merge?

The authors thank S. Patiri, I. Trujillo, D. Bettoni, and the referee for useful comments. A. V. and V. Q. are Ramón y Cajal Fellows of the Spanish Ministry of Education and Science. This work has been supported by the Spanish Ministry of Education and Science (grants AYA2004-03059 and AYA2004-08251-C02-01) and the Generalitat Valenciana (grant GV2005/244).

\section{REFERENCES}

Barnes, J. E. 2002, MNRAS, 333, 481

Baugh, C., Cole, S., Frenk, C., \& Lacey, C. 1998, ApJ, 498, 504

Beers, T. C., Huchra, J. P., \& Geller, M. J. 1983, ApJ, 264, 356

Bergmann, M. 2002, Ph.D. thesis, Univ. Texas, Austin

Bottema, R. 1988, A\&A, 197, 105

Cardiel, N., Gorgas, J., Cenarro, J., \& González, J. J. 1998, A\&AS, 127, 597

Carretero, C., Vazdekis, A., Beckman, J. E., Sánchez-Blázquez, P., \& Gorgas, J. 2004, ApJ, 609, L45

Clemens, M. S., Bressan, A., Nikolic, B., Alexander, P., Annibali, F., \& Rampazzo, R. 2006, MNRAS, 370, 702

Colbert, J., Mulchaey, J., \& Zabludoff, A. 2001, AJ, 121, 808

Collobert, M., Sarzi, M., Davies, R. L., Kuntschner, H., \& Colless, M. 2006, MNRAS, 370, 1213

Davies, R., Sadler, E., \& Peletier, R. 1993, MNRAS, 262, 650

de Lucia, G., Springel, V., White, S. D. M., Croton, D., \& Kauffmann, G. 2006, MNRAS, 366, 499

Djorgovski, S., \& Davis, M. 1987, ApJ, 313, 59

D'Onghia, E., et al. 2005, ApJ, 630, L109

Faber, S. M., \& Jackson, R. E. 1976, ApJ, 204, 668

Fisher, D., Franx, M., \& Illingworth, G. 1995, ApJ, 448, 119

González-García, A. C., \& van Albada, T. 2005, MNRAS, 361, 1043

Hernquist, L., \& Quinn, P. J. 1987, ApJ, 312, 1
Jaffe, W. 1983, MNRAS, 202, 995

Jones, L., Ponman, T., \& Forbes, D. 2000, MNRAS, 312, 139

King, I. R. 1966, AJ, 71, 276

Kobayashi, C., \& Arimoto, N. 1999, ApJ, 527, 573

Kormendy, J. 1977, ApJ, 218, 333

Kuijken, K., \& Dubinski, J. 1995, MNRAS, 277, 1341

Malin, D. F., \& Carter, D. 1983, ApJ, 274, 534

Pahre, M., Djorgovski, S., \& de Carvalho, R. 1998, AJ, 116, 1591

Peng, C. Y., Ho, L. C., Impey, C. D., \& Rix, H.-W. 2002, AJ, 124, 226

Quinn, P. J. 1984, ApJ, 279, 596

Springel, V., White, S. D. M., Tormen, G., \& Kauffmann, G. 2001, MNRAS, 328,726

Terlevich, R. J., Davies, R. L., Faber, S. M., \& Burstein, D. 1981, MNRAS, 196,381

Tonry, J., \& Davis, M. 1979, AJ, 84, 1511

Trujillo, I., Aguerri, A., Gutiérrez, C., \& Cepa, J. 2001, AJ, 122, 38

Vazdekis, A. 1999, ApJ, 513, 224

Weil, M., \& Hernquist, L. 1996, ApJ, 460, 101

Worthey, G., Faber, S. M., González, J. J., \& Burstein, D. 1994, ApJS, 94, 687

Zimmermann, H.-U., Boller, Th., Dobereiner, S., \& Pietsch, W. 2001, A\&A, 378, 30 